\title{
On keeping an open mind
}

\author{
Fiona Godlee editor, BMJ
}

\begin{abstract}
At some time or other, all of us will have fallen into the trap of "confirmation bias." This is the tendency to favour information that confirms our view, regardless of whether the information is true. In medicine this can be dangerous, as Ron Berghmans shows in his Patient Journey (doi:10.1136/bmj.d5469). Suffering from back pain, he consulted his GP on several occasions over a period of months, to be told that there was nothing seriously wrong and denied (within the Dutch health system) referral to a specialist. Experiencing increasing pain and disability, he eventually referred himself to a neurologist outside the Netherlands, and was found to have extensive non-Hodgkin's lymphoma.
\end{abstract}

Reading the story, you might conclude simply that this is a single unfortunate case in which one doctor failed to make a diagnosis. Berghmans uses his story to highlight a more general point. There are risks involved in doctors sticking to their preconceptions despite evidence to the contrary. He concludes that medical schools don't pay enough attention to the philosophy of science, and particularly to Karl Popper's falsification principle. This requires the formulation of testable hypotheses and the intention to falsify them. Do not try to look for white swans, says Popper, look for black swans instead. Berghmans wants to see a transformation in doctors' mindsets so that instead of seeking support for their convictions, they search for alternative explanations for their patients' complaints.

Confirmation bias is a trap for editors too. It's always easier to feel sympathetic towards a view that fits our own. So when deciding what to publish we have to remember to work harder to apply scepticism and scrutiny where an author's views are in tune with our own. I have no doubt that we don't always succeed. However here are two examples where I think we have succeeded.

The first led to last week's announcement by England's cancer tsar, Mike Richards, that there will be an inquiry into the benefits and harms of breast cancer screening (BMJ 2011;343:d6843). Over a period of years the $B M J$ has published articles raising concerns about the accuracy and transparency of information provided to women invited to attend screening. The $B M J$ was criticised, usually in emails not for publication, for damaging the UK's screening programme and for failing to put across the other side. So we asked for an analysis from someone we thought both sides would trust. In his article, Klim McPherson concluded that the evidence did not support the government's approach (BMJ 2010;340:c3106).

A second example is published this week. Controversy has been growing over whether the government's plans to increase competition will improve the quality of healthcare in England. Faced with requests from both sides of the argument to publish their views, we instead asked Gwyn Bevan and Matthew Skellern from the London School of Economics to review the evidence (doi:10.1136/bmj.d6470). As with breast screening, it's a complicated picture, with different time periods and methodologies and uncertainty over outcome measures. Their conclusion: patient choice should be introduced as an end in itself, but the question of whether competition improves quality remains open. So must our minds.

Cite this as: BMJ 2011;343:d7125

๑ BMJ Publishing Group Ltd 2011 